\title{
A Portable, Low-cost System for Optical Explosive Detection based on a CMOS Camera
}

\author{
Ross N. Gillanders ${ }^{1}$, Iain A. Campbell ${ }^{1}$, Fei Chen ${ }^{2}$, Paulina O. Morawska ${ }^{1}$, Ifor D. W. Samuel ${ }^{1}$ \\ and Graham A. Turnbull ${ }^{1}$ \\ ${ }^{1}$ Organic Semiconductor Centre, SUPA, School of Physics \& Astronomy, University of St Andrews, \\ Fife KY16 9SS, Scotland \\ ${ }^{2}$ Key Laboratory for Flexible Electronics, Institute of Advanced Materials (IAM), Nanjing Tech University, \\ 30 South PuZhu Road, NanJing, 211816, P. R. China
}

Keywords: Explosives, Conjugated Polymer, Organic Semiconductors, Raspberry Pi, Imaging.

\begin{abstract}
Humanitarian demining requires a variety of methods and instrumentation for effective mine clearance, since a wide range of materials are used in mine manufacturing. However, landmines release vapours over time that can be detected, for example, by sniffer dogs. Optical sensor systems are especially suited to this application due to the potential for lightweight, portable, low-cost systems that nevertheless have fast response times and ppb-level sensitivity to explosive vapours. In this paper we present a system for detection based on a low-cost Raspberry Pi platform with an integrated CMOS camera. The conjugated polymers Super Yellow and Polyfluorene are excited by an LED, and the quenching effect by DNB vapour is monitored by the camera to indicate the presence of explosives. The system shows potential as a userfriendly, lightweight platform for explosive vapour sensing.
\end{abstract}

\section{INTRODUCTION}

The tools required for humanitarian demining must be able to operate across a wide range of environments, since legacy landmines remain in countries with extremely disparate climates. These climates can range from Mediterranean, such as that in Croatia; to dry desert climates in Africa; to monsoon-prone countries, for instance those in South East Asia. Different tools for demining have different degrees of success in the various environments. The most common methods for mine detection include metal detectors and sniffer dogs; the combination of both is often used in the field. However, these methods can have disadvantages: metal detectors can identify harmless fragments of metal, or, with potentially fatal consequences, be unable to detect a mine made from another material such as plastic. Canines have strict working practices with the time the dogs can spend sniffing for mines restricted by both guidelines and the willingness of the dog at any particular moment (Porritt et al., 2015). Other common methods are inherently dangerous, such as prodders; thus there is a need in humanitarian demining for advanced, sensitive, robust instrumentation (Newnham and Daniels, 2001). In this paper we present a portable optoelectronic sensor based on explosive vapour detection.

Mines can release small amounts of vapour for a long time after being buried - for example, trinitrotoluene (TNT) degrades over time to produce dinitrotoluene (DNT), which then is released at ground level. There has been extensive work done on explosive sensing with conjugated polymers (Wang et al., 2011a; Wang et al., 2011b; Wang et al., 2012; Narayanan et al., 2008; Thomas et al., 2007), which are electron-rich materials used in organic semiconductor research. If nitroaromatic explosive vapours adsorb onto the polymer surface while these polymers are photoexcited by incident light, electrons are transferred to the deficient nitroaromatic vapour, resulting in loss of fluorescent emission, as shown in Figure 1. The luminescence can be monitored in real-time to provide a quenching profile, thus indicating the presence of explosive vapours.

One of the chief advantages to optical sensing is that it can be achieved through relatively low-cost, portable and fast-responding instrumentation. These 
factors are crucial in humanitarian demining, since the work is often conducted in challenging environments. Additionally, the operators of such devices will not necessarily be trained scientists or technicians, so ultimately the operation of the instrument should be user-friendly, with clear and distinctive data visualisation.
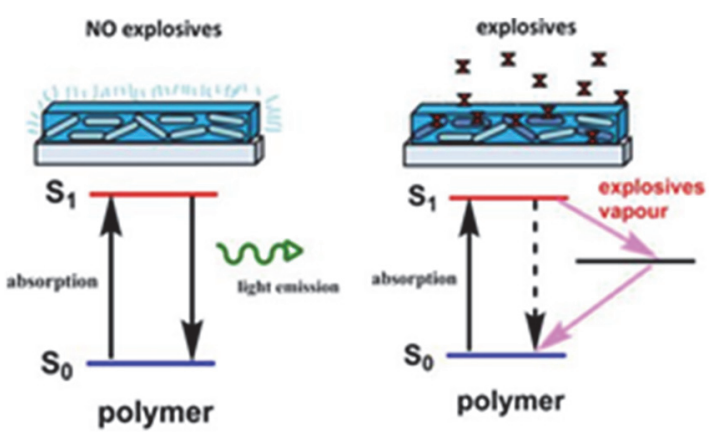

Figure 1: Quenching mechanism for conjugated polymer by explosive vapour.

While many fluorescence-based sensing platforms successfully utilise low-cost electronics, such as photodiodes, recent years have seen the introduction of off-the-shelf consumer computing platforms including the Arduino family of microprocessors and the Raspberry Pi. Use of these platforms in analytical instrumentation has gradually begun to appear in literature (Bitella et al., 2014; Tonacci et al., 2015; Leeuw et al., 2013), since they offer advantages in cost, open-source hardware and software, user-friendliness, small footprint, and flexibility in deployment. Smartphones have recently become tools for chemical sensors (Comina et al., 2015), and since the CMOS camera used in conjunction with the Raspberry Pi processor is similar to those found in typical smartphones, the technology presented here may eventually be integrated with them, broadening and making more accessible the system for communities in need of easy-to-use, low-cost, portable optical sensor systems for mine detection. While the change in luminescence intensity from the sensor films can be monitored using a standard silicon photodiode, this generally allows only the measurement of a single wavelength from a single film. The ability to measure multiple luminescent sensor spots with a single detector would present advantages in both simultaneous sensing of multiple analytes, and in selectivity to identify the, in this case, various nitroaromatic groups. The use of a camera system allows for this multi-analyte detection in the future since the principle of the image processing, reported here, can be applied to more than one emission spot in a single image. This system could prove useful in a "demining toolbox" in conjunction with other technologies to allow a deminer to make a choice of complementary systems appropriate for the environment. For instance, a metal detector could identify suspect sites, and the air sampled above those sites for trace explosive vapours. Since the system would also be suitable for sensing other analytes, it may be useful for environmental, food quality or other applications in the future.

In this paper we present an optical sensor system using the Raspberry Pi platform in conjunction with a CMOS camera, excitation LED, Polyfluorene (PFO) and Super Yellow (SY) conjugated polymers to detect dinitrobenzene (DNB) vapour. Early results indicate that the system could ultimately be applied for the detection of buried landmines.

\section{EXPERIMENTAL}

\subsection{Film Fabrication \& Characterisation}

Films based on Merck Super Yellow and PFO were prepared by spin-coating a $6.5 \mathrm{mg} / \mathrm{ml}$ solution of the polymer in toluene at $2000 \mathrm{rpm}$ onto a glass slide. The film thicknesses were measured with a Veeco Dektak 150 surface profiler and found to be $100 \mathrm{~nm}$ thick on average. Absorption and photoluminescence spectra were measured, respectively, using a Cary 300 Bio UV-Vis spectrometer and an Edinburgh Instruments FLS980 Fluorescence spectrometer. The peak absorption wavelengths for PFO and SY were $384 \mathrm{~nm}$ and $440 \mathrm{~nm}$ respectively. Peak emission wavelengths were $420 \mathrm{~nm}$ for PFO and $590 \mathrm{~nm}$ for SY. Photoluminescent Quantum Yield (PLQY) was measured with a Hamamatsu Photonics C9920-02 integrating sphere with excitation wavelengths of $384 \mathrm{~nm}$ for PFO and $440 \mathrm{~nm}$ for SY. The PLQYs of the polymers were measured to be $46 \%$ for PFO, and $40 \%$ for SY.

\subsection{Hardware}

The excitation LED for each polymer was chosen to match the absorption peak as closely as possible, with a Royal Blue LUXEON LED from Philips selected with a central wavelength of $447.5 \mathrm{~nm}$ for SY and a 370nm-centred LED from Thorlabs for the PFO excitation. The appropriate LED was aligned with the sample and detector as shown in Figure 2: the excitation light was filtered using a high-pass 
filter, and a neutral density filter of optical density 1 or 2 was inserted to reduce any saturation effects on the CMOS camera if necessary. The CMOS camera module from Raspberry Pi has 5 megapixels and a fixed focus.

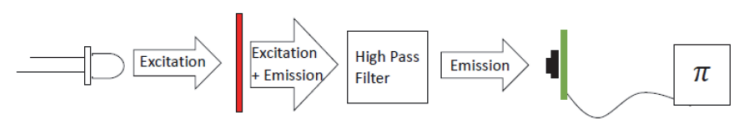

Figure 2: Hardware set-up schematic. A Royal Blue LUXEON LED excites the Super Yellow film, and the High-Pass filter removes the source light. The emitted light is collected by the camera and processed on-board the Raspberry Pi platform.

\subsection{Experimental Set-up}

The polymer-coated slide was inserted into an inhouse designed airtight chamber with gas flow inlet and outlet, and quartz windows on opposing sides for light excitation and emission. Powdered dinitrobenzene (Sigma Aldrich) was placed in a glass tube with a nitrogen line flowing through the powder, then via control valves leading to the chamber inlet. To make a measurement, the nitrogen flow was turned on and the luminescent emission monitored in real time with the camera. The images were taken at 20 second intervals. Measurements were conducted at room temperature.

\subsection{Image Processing}

The image processing was performed in Python using open source libraries from Raspberry Pi. Briefly, the system works by taking the original image, prior to binarization, i.e assigning "light" and "dark" regions to the image. Subsequently automatic circle detection defined the emission area, and finally automatic Region-of-Interest selection is used to define the monitored signal. The process is illustrated in Figure 3, with the decision-making flow-chart shown in Figure 4.

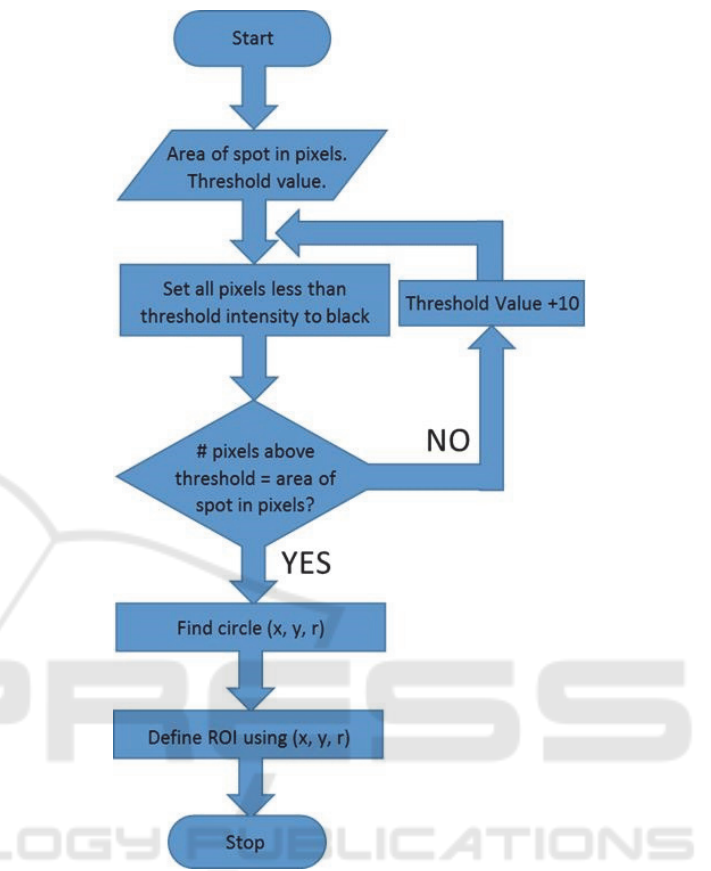

Figure 4: Flowchart for image processing.

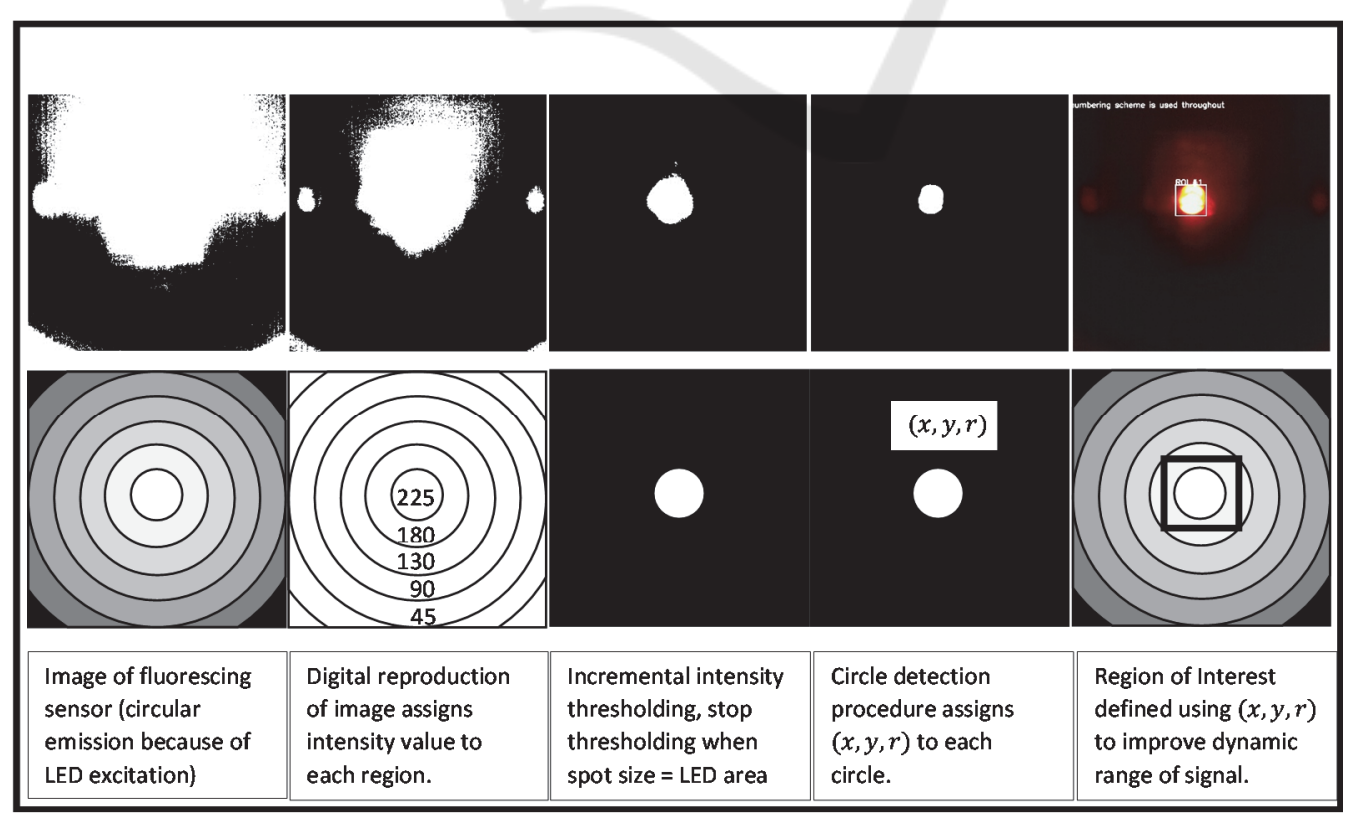

Figure 3: Image Processing with an illuminated Super Yellow film. 


\section{RESULTS}

Figure 5 shows the quenching profile of the Super Yellow film to DNB vapour at a vapour pressure of approximately $30 \mathrm{ppb}$ (Ewing et al., 2013). A clear and measurable decrease in luminescent intensity is shown, with the quenching effect apparent after the first 20 seconds. The intensity decreases by approximately $6 \%$ in 200 seconds.

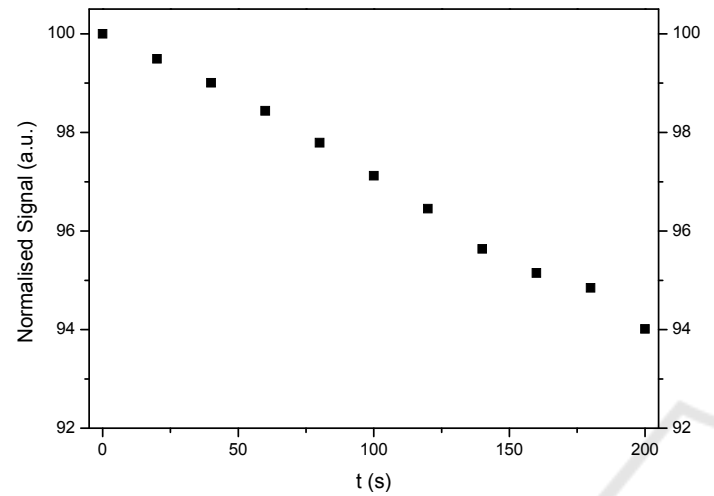

Figure 5: Quenching profile of Super Yellow exposed to DNB vapour in nitrogen.

The response of PFO to DNB is shown in Figure 6 . The quenching response is slightly lower than that of SY at around $4.5 \%$.

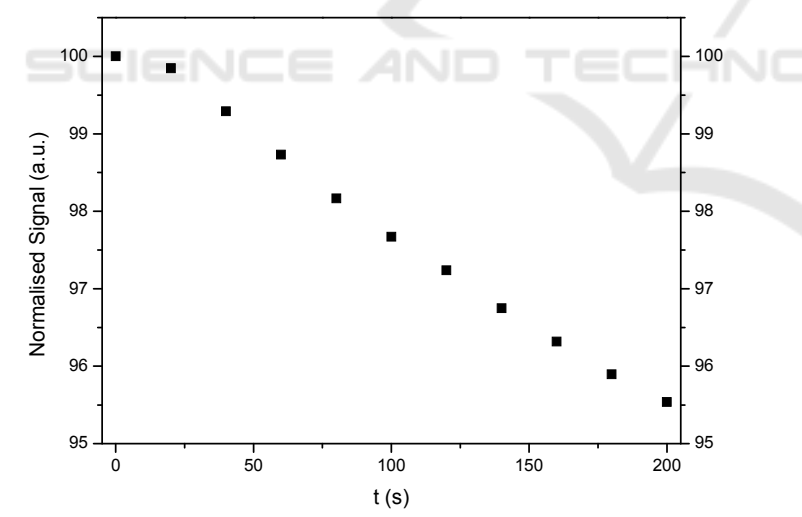

Figure 6: Quenching profile of PFO exposed to DNB vapour in nitrogen.

These results indicate that the CMOS camera system is a suitable method for optical detection of ppb-level explosive vapours. The inherent difference between the two polymers' response to the DNB vapour suggests that both could be monitored simultaneously, leading to selective measurements for identification of explosive vapours. The system reported here is lightweight, inexpensive and lowcost in addition to its sensitivity, so further work is ongoing to ruggedize the system for field trials, build a library of polymer-vapour response curves, and investigate simultaneous multi-analyte sensing.

\section{CONCLUSIONS}

An optical sensing system was developed using an off-the-shelf Raspberry Pi processing platform in conjunction with a CMOS camera, using the conjugated polymers Super Yellow and Polyfluorene as the explosive vapour-sensitive sensor films. The quenching effect of the vapour on the polymers' luminescence was successfully monitored by the camera and profiles generated over around 200 seconds of vapour adsorption to the polymer surface. The system shows promise to be used as a portable, user-friendly explosive vapour sensor in humanitarian demining, and in this be useful complementing other methods such as metal detectors to more thoroughly detect and identify buried landmines. Improvements and further work to the system have been identified towards development of a robust, highly-sensitive instrument.

\section{ACKNOWLEDGEMENTS}

This project has received funding from the European Union's Seventh Framework Programme for research, technological development and demonstration under agreement no 284747.

\section{REFERENCES}

Bitella, G., Rossi, R., Bochicchio, R., Perniola, M. \& Amato, M. 2014. A Novel Low-Cost Open-Hardware Platform for Monitoring Soil Water Content and Multiple Soil-Air-Vegetation Parameters. Sensors, 14, 19639-19659.

Comina, G., Suska, A. \& Filippini, D. 2015. Autonomous Chemical Sensing Interface for Universal Cell Phone Readout. Angewandte Chemie-International Edition, 54, 8708-8712.

Ewing, R. G., Waltman, M. J., Atkinson, D. A., Grate, J. W. \& Hotchkiss, P. J. 2013. The Vapor Pressures of Explosives. Trac-Trends in Analytical Chemistry, 42, 35-48.

Leeuw, T., Boss, E. S. \& Wright, D. L. 2013. In Situ Measurements of Phytoplankton Fluorescence using Low Cost Electronics. Sensors, 13, 7872-7883.

Narayanan, A., Varnavski, O. P., Swager, T. M. \& Goodson, T., Iii 2008. Multiphoton Fluorescence 
Quenching of Conjugated Polymers for Tnt Detection. Journal of Physical Chemistry C, 112, 881-884.

Newnham, P. \& Daniels, D. 2001. The Market for Advanced Humanitarian Mine Detectors. Conference on Detection and Remediation Technologies for Mines And Minelike Targets Vi, 2001.

Apr 16-20 2001 Orlando, Fl. 1213-1224.

Porritt, F., Shapiro, M., Waggoner, P., Mitchell, E., Thomson, T., Nicklin, S. \& Kacelnik, A. 2015. Performance Decline by Search Dogs in Repetitive Tasks, and Mitigation Strategies. Applied Animal Behaviour Science, 166, 112-122.

Thomas, S. W., Iii, Joly, G. D. \& Swager, T. M. 2007. Chemical Sensors based on Amplifying Fluorescent Conjugated Polymers. Chemical Reviews, 107, 13391386.

Tonacci, A., Corda, D., Tartarisco, G., Pioggia, G. \& Domenici, C. 2015. A Smart Sensor System for Detecting Hydrocarbon Volatile Organic Compounds in Sea Water. Clean-Soil Air Water, 43, 147-152.

Wang, Y., Mckeown, N. B., Msayib, K. J., Turnbull, G. A. \& Samuel, I. D. W. 2011a. Laser Chemosensor with Rapid Responsivity and Inherent Memory based on a Polymer of Intrinsic Microporosity. Sensors, 11, 24782487.

Wang, Y., Turnbull, G. A. \& Samuel, I. D. W. 2011 b. Conjugated Polymer Sensors for Explosive Vapor Detection. Organic Semiconductors in Sensors and Bioelectronics $I v, 8118$.

Wang, Y., Yang, Y., Turnbull, G. A. \& Samuel, I. D. W 2012. Explosive Sensing Using Polymer Lasers. Molecular Crystals and Liquid Crystals, 554, 103-110. 